\title{
Combination of Spherical and Planar Scanning for Phaseless Near-Field Antenna Measurements
}

\author{
Fernando Rodríguez Varela, Belén Galocha Iraguen, \\ Manuel Sierra Castañer
}

\author{
Javier Fernández Alvárez, Michael Mattes, \\ Olav Breinbjerg
}

\begin{abstract}
The two scans phaseless technique is a well-known procedure for the characterization of antennas on near-field ranges without need of measuring the phase. Amplitude information over two surfaces compensates for the lack of phase reference. In this paper we propose the combination of spherical and planar surfaces for the application of the two scans technique, together with the application of Wirtinger Flow, a state-of-the art phase retrieval algorithm with high convergence guarantees. The use of different types of surface adds additional information about the field's degrees of freedom, allowing for smaller separation between acquisition surfaces as compared with the 2-sphere techniques. In addition, an initial estimation for the phase is not required. The phase retrieval process is formulated in terms of the Spherical Wave Expansion (SWE) of the antenna under test. The SWE-to-PWE (Plane Wave Expansion) is utilized in order to process the amplitude field on the planar surface. Results for simulated and measured near-field data are shown to demonstrate the potential capabilities of the proposed technique.
\end{abstract}

\section{INTRODUCTION}

Near-field measurements [1] constitute an accurate and powerful set of techniques for antenna characterization. Performing the measurements in the near-field region enables the use of relatively small anechoic chambers in exchange of a mathematical transformation that can be efficiently implemented with the current processing power and developed techniques. A major challenge in antenna measurements is the accurate characterization of phase at high frequencies, which can become unstable due to cable movement, drifts and small deviations of the positioning equipment. In other cases, phase information cannot be obtained at all, as in the case of devices with embedded RF sources from which the phase reference cannot be extracted, or when the presence of a cable to transmit this reference to the VNA is impractical, as in the case of aerial antenna measurements.

Therefore, the antenna community has been working on phaseless techniques in order to perform antenna measurements and post processing without any need of phase reference. The main techniques are the holographic [2], the interferometric [3][4] and the two scans technique [5]-[9]. The two first are able to extract the near-field phase with a simple post processing but require the use of special measurement equipment that must be calibrated, resulting in a new challenging task. On the other hand, the two scans technique can recover the phase information with the same equipment used for traditional complex measurements, and it shows improved robustness against electrical and mechanical inaccuracies which are critical at high frequencies.

The two scans technique is based on a pair of magnitude measurements performed at different distances from the antenna under test (AUT). Amplitude information over two different distances adds enough information to compensate for the lack of phase. This has been applied for the spherical [5][6] and planar [7][8] case. By measuring the amplitude on two separate planes or spheres, the phase can be retrieved solving a nonlinear minimization problem. This can be solved using iterative propagation techniques [5]-[8] or a direct optimization algorithm [9].

When working with nonlinear problems, non-uniqueness and local minima issues arise. Although it has not been demonstrated, uniqueness of the solution when measuring the fields on two planes or two spheres is strongly suspected [10]. Despite, finding this unique solution has proven to be a quite challenging computational and mathematical task. The nonlinear nature of the problem makes it prone to get trapped in local minima and suboptimal solutions. To avoid this, some of the presented techniques rely on the choice of a judicious initial guess for the phase and in the increase of the two surfaces separation. A large separation allows the field to propagate and experience variations in amplitude, which increases the information added to the problem. In practice, it has been observed that a considerable distance is required for the fields to experiment meaningful changes in the amplitude [5]. These distances can result in impractical measurement configurations requiring large translation stages and anechoic chambers.

In this paper the combination of a planar scanning and a spherical scanning for phaseless measurements is proposed (see Figure 1). The projection of the fields over two different geometries increases the independence between the two scans, so the presence of local minima can be mitigated with smaller separation distances than in the case of the two spheres scan technique. This allows to solve phase retrieval problems with less dependency on the initial guess, which has proven to be a challenging issue on the two scans planar technique [8]. The amplitude measurements over a plane and a sphere are supplied to a state-of-the-art phase retrieval minimization algorithm that 


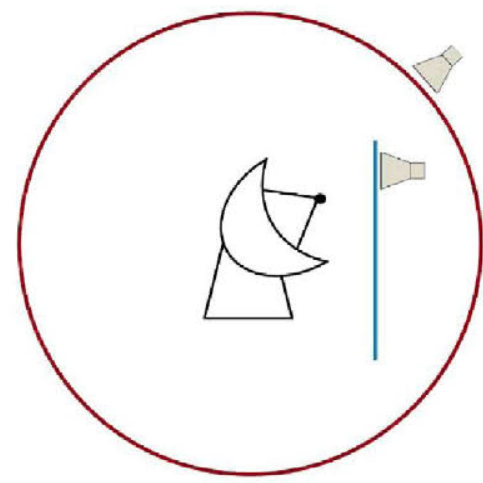

Figure 1. Schematic representation of the proposed measurement configuration

obtains the spherical wave expansion (SWE) of the AUT in an efficient way.

In particular, the proposed technique can be implemented in anechoic chambers where the AUT sits in a roll over azimuth positioner and the probe can perform a planar scanning. A translation stage is also required to vary the distance between AUT and probe, as in the 2 -spheres technique. If the positioners do not allow such displacement, the AUT can also be measured in a conventional spherical range and then moved to a planar set up. This offers a way of performing phaseless measurements in scenarios in which it would be impossible to apply the 2 -spheres technique.

The organization of this paper is as follows. In Section II the details of this formulation will be disclosed. Section III will present some simulation results. Finally, transformation results of antennas measured in an anechoic chamber will be shown in Section IV.

\section{THEORETICAL BACKGROUND}

Fields radiated by the AUT can be represented by the wellknown spherical wave expansion:

$$
\vec{E}(r, \theta, \varphi)=\sum_{s=1}^{2} \sum_{n=1}^{N} \sum_{m=-n}^{n} Q_{s m n} \vec{F}_{s m n}^{(3)}(r, \theta, \varphi)
$$

$(r, \theta, \varphi)$ being spherical coordinates, $Q_{s m n}$ the spherical wave coefficients (SWC) and $\vec{F}_{s m n}^{(3)}$ the spherical wave basis functions. $N$ is the truncation number of the expansion, and its value depends on the electrical size of the AUT:

$$
N=\left\lceil k a_{l}\right\rceil+10
$$

where $a_{l}$ is the radius of the minimum sphere circumscribing the AUT, $k$ the free space wavenumber, and the brackets indicate the largest integer smaller than or equal to the number inside them.

On the proposed method, the SWC will be the unknows to be found from the knowledge of the field amplitude on a sphere and plane. Therefore, we must derive analytical expressions that give the interaction between the SWC and the field measured by the probe in points over a plane and sphere.

\section{A. AUT-probe interactions on spherical surface}

The well-known transmission formula [11] offers an analytical expression to compute interactions between the AUT and the probe over a spherical scanning in terms of the SWC of both antennas:

$$
\begin{aligned}
& m_{s p h}(r, \theta, \varphi, \chi) \\
& =\sum_{\sigma \mu \nu}^{s m n} Q_{s m n} R_{\sigma \mu \nu} e^{j m \varphi} d_{\mu m}^{n}(\theta) e^{j \mu \chi} C_{\sigma \mu \nu}^{s n}(k r)
\end{aligned}
$$

being $R_{\sigma \mu \nu}$ the probe SWC coefficients and $d_{\mu m}^{n}$ and $C_{\sigma \mu \nu}^{s n}$ some rotation and translation terms. In this case, it is convenient to work with the discrete matrix-vector representation of (5):

$$
\vec{m}_{s p h}=C_{s p h} \vec{q}
$$

in which $C_{s p h}$ performs the required summations and multiplications and $\vec{m}_{s p h}$ and $\vec{q}$ are vector containing the measured samples and $\mathrm{SWC}$, respectively

\section{B. AUT-probe interactions on planar surface}

In principle, the SWE can be evaluated at arbitrary points in the space taking into account the probe effect. However, (5) is only valid outside the minimum sphere, which makes it impossible to model scenarios where the probe is scanning very close to the AUT, a typical configuration in planar measurements. In addition, extra rotation terms would be needed to account for the probe radiation pattern effect because (5) assumes that the probe is always pointing to the AUT. Therefore, we propose to use instead the Plane Wave Spectrum (PWS) representation of the AUT, which is the natural basis to compute antenna interactions over planar geometries. This process requires following a series of steps.

First, we introduce the SWE-to-PWE (Plane Wave Expansion) [11] transformation which allows to calculate the PWS of the AUT from the SWC:

$$
\vec{T}(\hat{k})=\sum_{s=1}^{2} \sum_{n=1}^{N} \sum_{m=-n}^{n} Q_{s m n} \vec{Y}_{s m n}(\hat{k})
$$

where $\vec{Y}_{s m n}$ are the vector spherical harmonics defined in [11] and $\vec{T}(\hat{k})$ the PWS. As in the previous case, we work with the matrix version of (7):

$$
\vec{t}=C_{\text {swe_pwe }} \vec{q}
$$

Once the AUT PWS is known, the interaction with the probe can be computed straightforwardly [13]:

$$
m(\vec{r})=\int_{\infty}^{\infty} \vec{T}(\hat{k}) \cdot \vec{P}(\hat{k}) e^{-j \hat{k} \vec{r}} d \hat{k}
$$

and its matrix-vector form:

$$
\vec{m}_{p l}=C_{p w e \_p l n} \vec{t}
$$

Finally, (8) and (10) are combined to obtain a closed form expression for the interaction between AUT and probe:

$$
\vec{m}_{p l}=C_{p w e \_p l n} C_{\text {swe_pwe }} \vec{q}=C_{p l n} \vec{q}
$$




\section{Complete Phaseless problem}

With the analytical expressions linking the AUT SWC and the measured field over the two surfaces, the total interaction is given by:

$$
\left(\begin{array}{c}
\vec{m}_{s p h} \\
\vec{m}_{p l}
\end{array}\right)=\left(\begin{array}{c}
C_{s p h} \\
C_{p l n}
\end{array}\right) \vec{q} \rightarrow \vec{m}_{\text {total }}=C_{\text {total }} \vec{q}
$$

In the case of phaseless measurements, only amplitude information is available: $\left|\vec{m}_{\text {total }}\right|$. Then the phaseless problem can be formulated as:

$$
\left|\vec{m}_{\text {total }}\right|=\left|C_{\text {total }} \vec{q}\right|
$$

which will be solved using Wirtinger Flow [14], a non-convex phase retrieval solver. It is based on an initialization obtained by means of a spectral method and a series of updates refining this initial estimate by iteratively applying update rules, following a gradient descent scheme based on Wirtinger derivatives.

Wirtinger Flow offers convergence guaranties if the rows of $C_{\text {total }}$ are independently distributed and drawn from a complex normal distribution, and if there is some degree of oversampling. This is a purely mathematical condition not achievable in this case because matrix $C_{\text {total }}$ is built from the spherical and planar vector wave functions. We only have control over the measurement points, and these are restricted to locations which can be performed in practical measurement set ups. This means that there will exist some level of redundancy between the measurements, so the algorithm may fall in local minima. Therefore, it is crucial to increase the amount of independent information, which can be achieved by taking measurements over two well separated surfaces or two different, as proposed in this paper.

\section{SimUlation RESUltS}

This section demonstrates the functionality of the proposed approach to recover the phase and far-field pattern from two near-field scannings over a plane and a sphere. This will be done using a simulation model based on Huygens sources (crossed magnetic and electric dipoles). This scenario will model the anechoic chamber and the electrical dimensions of the antennas that will be shown latter on section IV. The AUT is an array of size $17 \times 17 \mathrm{~cm}^{2}$ with $8 \times 8$ elements separated $0.7 \lambda$ fed with uniform amplitude and phase distribution at $10 \mathrm{GHz}$. The probe is another Hertz dipole which measures the electrical field over two surfaces:

- A plane of $57 \times 57 \mathrm{~cm}^{2}$ located at $14 \mathrm{~cm}$ from the AUT aperture (yielding a reliable angle of $55^{\circ}$ ) with measurements taken on an uniform grid at a sampling rate of $0.39 \lambda(60 \times 60$ samples).

- A sphere of radius $33 \mathrm{~cm}$ scanned with a spiral sampling of 60 turns with 3600 equispaced points in total. This sampling scheme allows for faster acquisition than the conventional equiangular one and has proven to mitigate the redundancy.

At each measurement point, the probe is rotated $90^{\circ}$ to obtain information of both polarizations.
With the defined scenario, the measured field amplitudes are simulated and the coupling matrix is constructed. This data is supplied to Wirtinger Flow, which will attempt to solve the phase retrieval problem. It is stressed that no additional information like initial guess is given to the solver. Instead, Wirtinger Flow will make an estimation based on a spectral analysis method. At each iteration of the algorithm, the amplitude error is computed as follows:

$$
\epsilon_{\text {amp }}(l)=\frac{r m s\left(\left|\vec{m}_{\text {total }}\right|-\left|C_{\text {total }} \vec{q}_{l}\right|\right)}{\max \left(\left|\vec{m}_{\text {total }}\right|\right)}
$$

$\vec{q}_{l}$ being the retrieved SWC at the $l$ th iteration. This error metric only gives information about how well the amplitude is reconstructed, and it is the only one available for a real phaseless problem. In this simulation scenario, we have information of the AUT, so we can compute the complex error as follows:

$$
\epsilon_{\text {comp }}(l)=\frac{r m s\left(\vec{q}_{l}-\vec{q}_{r e f}\right)}{\max \left(\left|\vec{q}_{r e f}\right|\right)}
$$

where $\vec{q}_{l}$ is the true SWC of the AUT. This figure of merit is not available in a real phaseless measurement scenario for obvious reasons, but it will be used here to gain further insight into the algorithm capabilities.

Figure 2 depicts the evolution of these error metrics with the iteration number. The amplitude error achieves a very low value which means that the algorithm has found a set of SWC radiating a field that matches the measured amplitude down to a level of around $-60 \mathrm{~dB}$. However, the complex error saturates at a level of $-32 \mathrm{~dB}$ so this will be the error of the retrieved solution. The far-field of the retrieved SWC is evaluated, and the obtained radiation pattern has been depicted in Figure 3. For comparison, the transformed far-field using both amplitude and phase information has also been calculated and displayed, obtaining an error of $-30 \mathrm{~dB}$.

The source of this discrepancies has been investigated by comparing the retrieved SWC with the reference. Figure 4 depicts a comparison of the $\mathrm{N}$-mode power spectrum of the

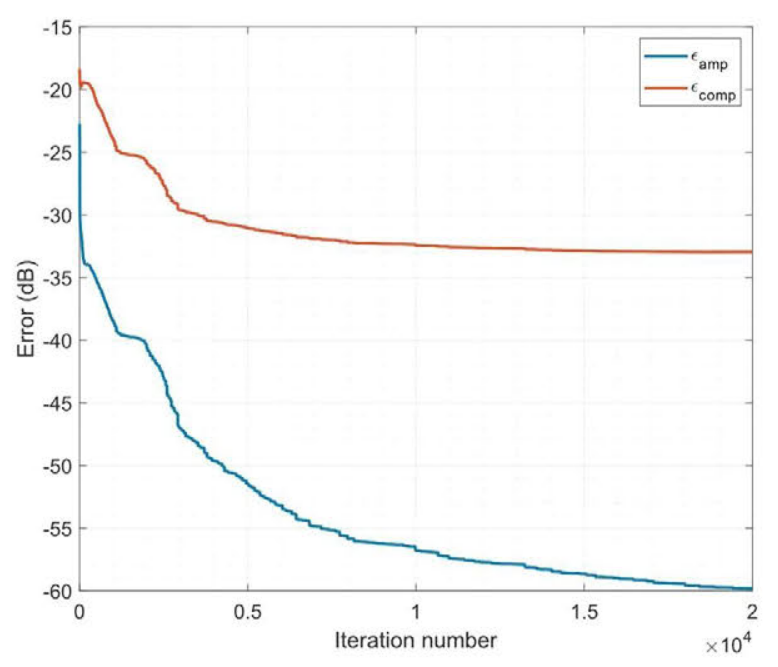

Figure 2. Evolution of the complex and amplitude error with the iteration number 


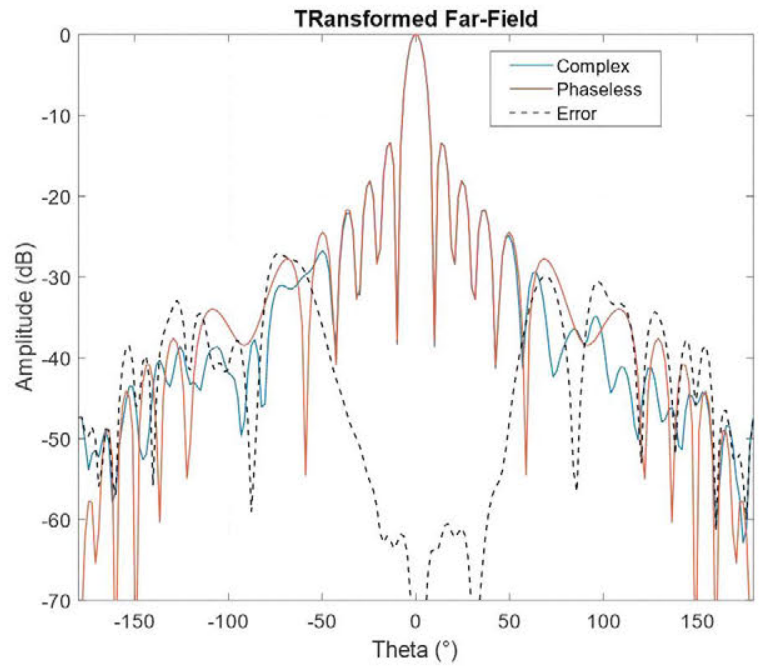

Figure 3. Comparison of the phaseless and complex far-field transformation for the array

phaseless solution compared with the references. The N-power spectrum of the difference between the two SWE has also been depicted. It can be seen that all harmonics show a similar level of discrepancy, though there exists a small tendency to increase with the degree of the harmonic. This may be due to the fact that slow variations are better sampled and can be retrieved with better accuracy.

In addition, the retrieved phase on the measurement surfaces has been depicted in Figure 5. It can be seen how the phase on the plane is virtually identical to the reference. However, in the case of the sphere, the far-lobes are retrieved with important deviations. These deviations are reflected in the far-field. The main reason for this discrepancy is the plane truncation. For values of $\theta>55^{\circ}$ the field has only been measured on the sphere, so the algorithm has to retrieve the phase using information of only this surface.

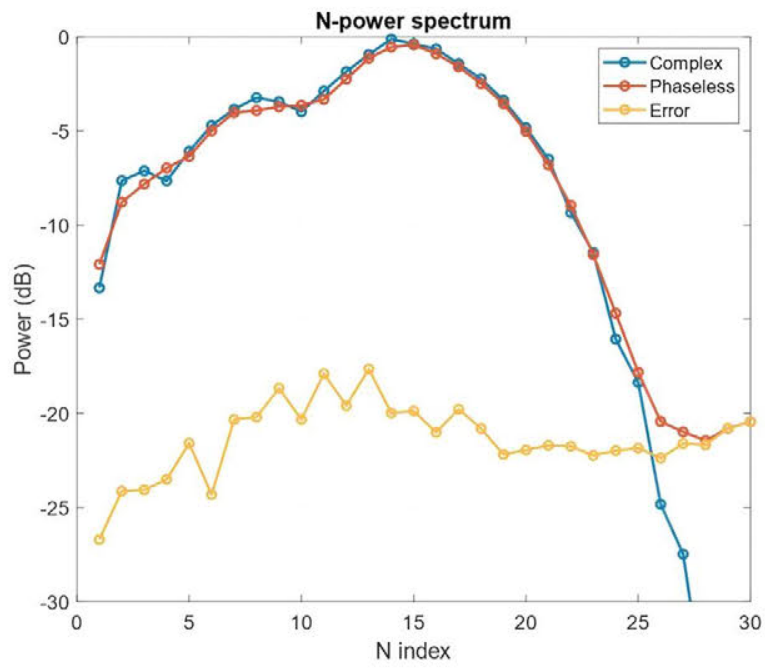

Figure 4. Comparison of the phaseless and complex $\mathrm{N}$-power spectrum retrieved
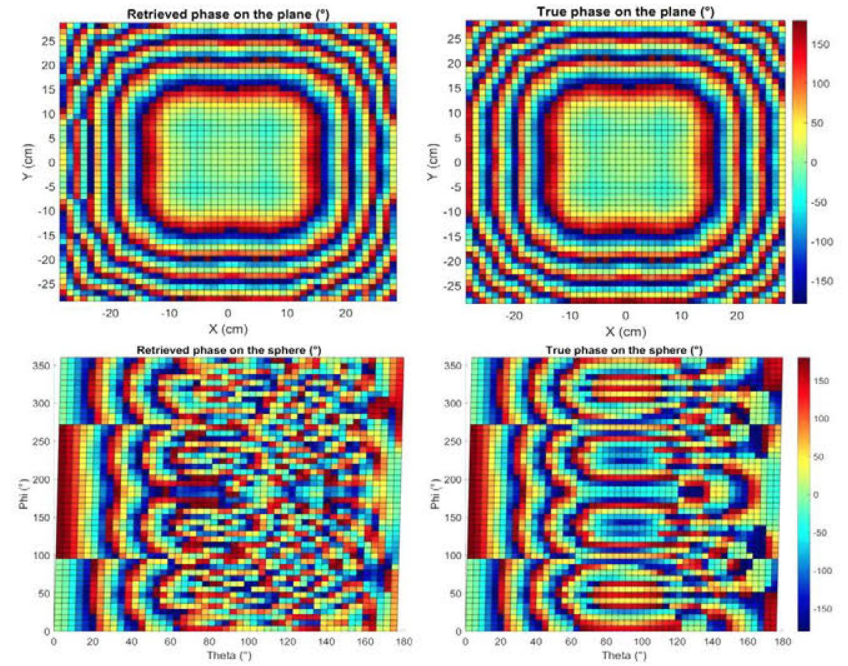

Figure 5. Comparison of the retrieved (left) and reference (right) phase on the measurement surfaces: plane (top) and sphere (bottom)

\section{A. Phase retrieval of a steered antenna}

In some publications [8] it has been pointed out the challenge of performing phaseless measurements of steered beam antennas. When an AUT is pointing in the boresight direction, the phase distributions possess some properties of symmetry and flatness that simplify the phase retrieval process. If a tilt angle is introduced, the phase distribution is significantly modified, and the phase retrieval algorithm is prone to be trapped in local minima. In [8] this issue is addressed by performing a global search of different tilt angles as initial guess for the minimization problem. Here we will show the robustness of the proposed phaseless technique by retrieving the phase of a beam-steered antenna without any initial guess or global search.

The measurement scenario of the last section is reused here. Now, we apply an incremental phase shift to the dipole array along the different columns in order to obtain a steering angle of $20^{\circ}$ in the radiation pattern. The size of the plane is enlarged to $70 \times 70 \mathrm{~cm}$ in order to reduce the truncation effect. The fields on the plane and sphere are simulated under these conditions and the Wirtinger Flow is started.

Figure 6 depicts the transformation results of the phaseless problem compared to the one obtained from a complex measurement. As it can be seen, the fields over the sphere and plane are able to incorporate enough information to allow the solver to converge to a solution with an error level around -30 $\mathrm{dB}$.

\section{B. Comparison with two-spheres technique}

The advantage of combining a spherical and planar scanning is that we increase the amount of non-redundant information as compared with the two spheres technique. When fields are projected over different surfaces, additional information about 


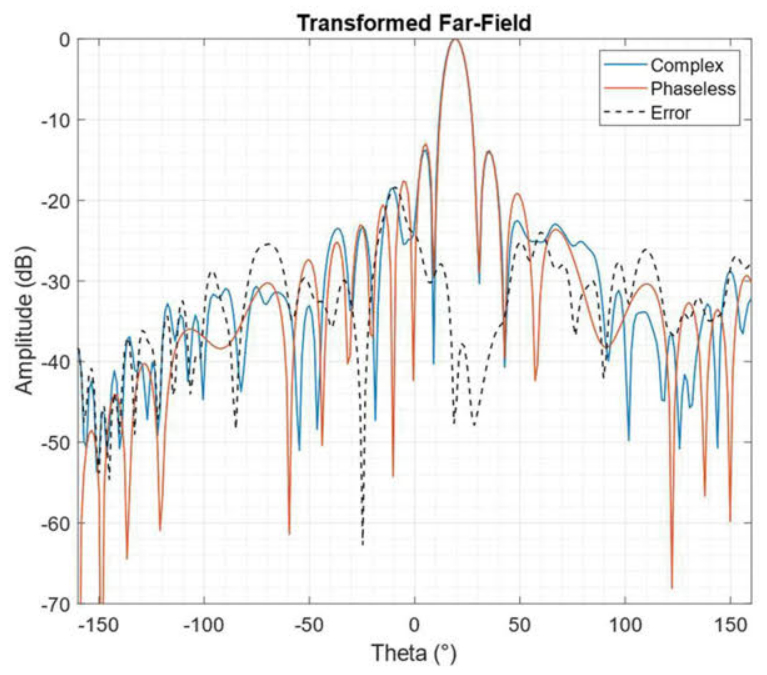

Figure 6. Comparison of the phaseless and complex transformed far-field for a steered beam antenna

the field degrees of freedom is gained that can be fundamental for the convergence of the problem. This will be shown with another simulation example in which different combinations of planes and spheres will be used to solve the phase retrieval problem using Wirtinger Flow.

We continue with the same dipole-based simulation scenario but in this case, the radius of the sphere is varied between $14 \mathrm{~cm}$ and $50 \mathrm{~cm}$, in order to observe how this parameter influences the retrieved solution. For each radius, the far-field radiation pattern is computed, and the relative error is calculated as follows:

$$
\begin{aligned}
& \varepsilon_{\text {far }} \\
& =10 \log _{10}\left(\frac{1}{N} \sum_{n=0}^{N} \frac{\left|E_{r e f}\left(\theta_{n}, \varphi_{n}\right)-E\left(\theta_{n}, \varphi_{n}\right)\right|^{2}}{\max \left|E\left(\theta_{n}, \varphi_{n}\right)\right|^{2}}\right)
\end{aligned}
$$

where $E\left(\theta_{n}, \varphi_{n}\right)$ is the calculated field for the given measurement sphere radius, and $E_{r e f}\left(\theta_{n}, \varphi_{n}\right)$ the reference field obtained from a complex measurement, both sampled on a grid of $N$ points. Figure 7 (black curve) depicts the evolution of this error with the different sphere radii. As the radius increases, the sphere and plane are separated, allowing the fields to propagate between the two surfaces. This increases the amount of independent information thus improving the retrieved solution.

A similar test is now performed for two spheres as measurement surfaces. One of the spheres is set at a fixed radius of $14 \mathrm{~cm}$, corresponding to the same value of distance between plane and AUT in the previous case. The radius of the other sphere is swept between 14 and $50 \mathrm{~cm}$, obtaining another error curve (red) that is also shown in Figure 7. In this case, the obtained errors are much higher. Up to values of $35 \mathrm{~cm}$, the algorithm gets trapped in a local minimum that gives an error of $-15 \mathrm{~dB}$. For higher values of separation, the two spherical surfaces are independent enough for the algorithm to escape these minima and progressively obtain a better solution. However, the obtained errors are higher than in the plane-sphere case.

In [5] it was observed that phaseless spherical measurements using small separation between probe and AUT give worse

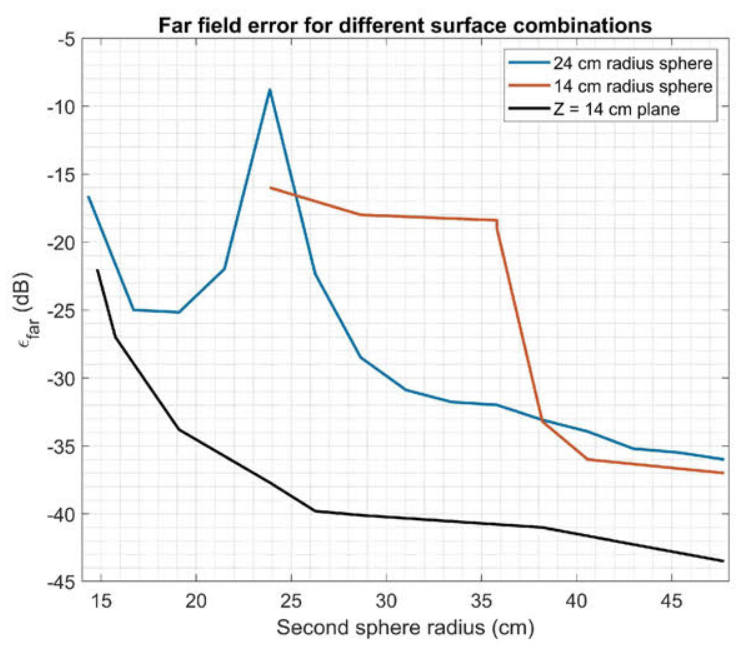

Figure 7. Evolution of the far-field error as a function of the sphere radius when the other surface is another sphere or a plane

results than those at intermediate distances. Therefore, the same radius sweep is performed but fixing one of the spheres at a radius of $24 \mathrm{~cm}$. The error results of this test are also depicted in Figure 7 (blue curve), where it can be seen that the overall errors have been reduced thanks to the greater separation between AUT and probe. This is confirmed by observing how the error eventually starts to increase as the second sphere gets close to the AUT. Even in this case, the obtained errors are higher than in the plane-sphere case. Therefore, we conclude that the use of a second planar surface instead of a sphere offers a way of obtaining independent information with smaller separations. This can relax the requirements for anechoic chamber dimensions or the movement range of the probe translation stage.

\section{MEASUREMENT RESULTS}

Finally, the proposed algorithm is applied to measured data, to assess its robustness against real noise, drift and other nonideal effects. The measurements have been performed at the anechoic chamber of the Technical University of Madrid (UPM). First the AUT field is measured in a spherical near-field facility acquiring both amplitude and phase. From this measurement, the SWE is obtained and then a planar measurement is simulated using the analytical formulation introduced in Section II.

The first test consists of a $2 \times 2$ patch array antenna measured using as probe a conical horn at $11 \mathrm{GHz}$. On the spherical surface, the distance between probe and antenna is $5 \mathrm{~m}$ and a spiral sampling of 70 turns with 4900 points with two polarizations is employed. This corresponds to an oversampling ratio of 1.36 with respect to a traditional complex measurement. The planar surface has a size of $57 \times 57 \mathrm{~cm}^{2}$ with a sampling rate of $0.22 \lambda$ and at a distance of $15 \mathrm{~cm}$ from the AUT. Overall, a total oversampling of 3.57 is taken with respect to a complex measurement on a single surface. Figure 8 shows the copolar and crosspolar patterns of a $\varphi=45^{\circ}$ cut. The transformation errors are quite small obtaining an agreement down to $-35 \mathrm{~dB}$. The second test is a $15 \mathrm{GHz}$ conical horn of spline profile measured 
on the same sphere of the previous case. The measurement plane has a dimension of $25 \times 25 \mathrm{~cm}^{2}$ and is placed at $7 \mathrm{~cm}$ from the horn aperture. The sampling rate is also $0.22 \lambda$. Figure 9 depicts the copolar and crosspolar patterns of a $\varphi=45^{\circ}$ cut. In this case there is good agreement down to a level of $-40 \mathrm{~dB}$ because the radiated power is more concentrated on the forward hemisphere and it can be measured on the plane.

\section{CONCLUSION}

A phase retrieval algorithm based on Wirtinger Flow and the combination of a spherical and planar scans has been proposed. A nonlinear minimization problem is formulated, where the unknowns are the SCW of the AUT, which are iteratively evaluated on the plane and the sphere until they match the measured amplitudes. For the evaluation on the sphere, the

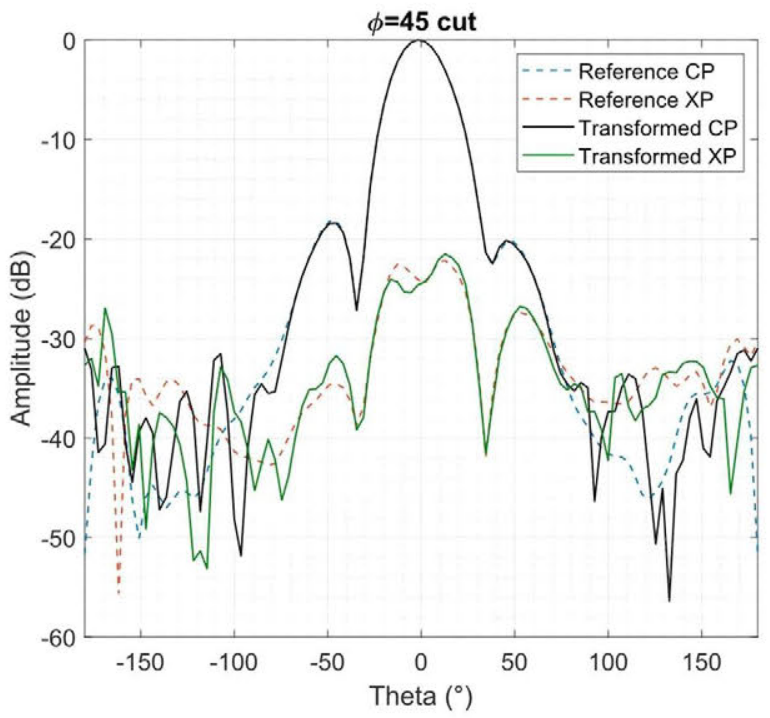

Figure 8. Comparison o complex and phaseless transformation for the patch antenna

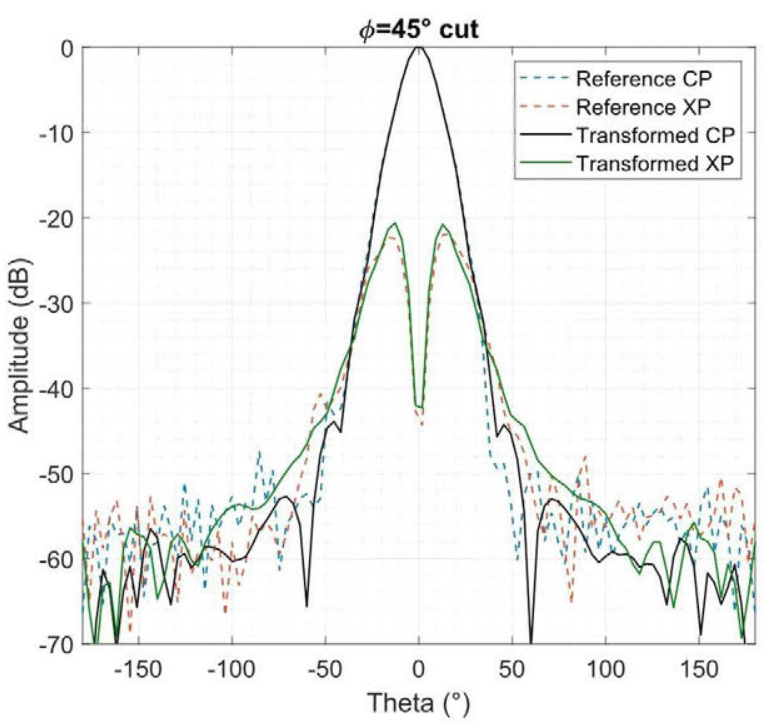

Figure 9. Comparison of complex and phaseless transformation for the horn antenna transmission formula for spherical waves is employed, as for the plane, the SWE-to-PWE transformation is used. Projection of the fields over two different surfaces adds valuable information to the problem, allowing to retrieve the phase with small separations between plane and sphere even in the case of steered beam antennas.

\section{ACKNOWLEDGEMENT}

This work was developed at the Department of Electrical Engineering of the Technical University of Denmark, and it has been supported by Universidad Politécnica de Madrid (Programa Propio) and by the Spanish Government, Ministry of Economy, National Program of Research, Development and Innovation through the project FUTURE-RADIO (project number TEC2017-85529-C3-1-R).

\section{REFERENCES}

[1] A. Yaghjian, "An overview of near-field antenna measurements," IEEE Transactions on Antennas and Propagation, vol. 34, no. 1, pp. 30-45, January 1986.

[2] J. Laviada, Y. Avarez-Lopez, A. Arboleya-Arboleya, C. Garcia-Gonzalez and F. Las-Heras, "Inverse scattering with phase retrieval based on indirect holography via synthesised plane-waves", IET Microwaves Antennas \& Propagation, vol. 6.12, pp. 1389, 2012.

[3] S. Costanzo, G. Di Massa and M. D. Migliore, "A novel hybrid approach for far-field characterization from near-field amplitude-only measurements on arbitrary scanning surfaces", IEEE Transactions on Antennas and Propagation, vol. 53, no. 6, pp. 1866-1874, 2005.

[4] A. Paulus, J. Knapp and T. F. Eibert, "Phaseless Near-Field Far-Field Transformation Utilizing Combinations of Probe Signals," IEEE Transactions on Antennas and Propagation, vol. 65, no. 10, pp. 54925502, Oct. 2017.

[5] J. Fernandez Alvarez, J. M. Bjørstorp, and O. Breinbjerg, (2018). "Spherical Phaseless Probe-Corrected Near-Field Measurements of the DTU-ESA VAST12 Reflector Antenna". Proceedings of the 40th Annual Symposium of the Antenna Measurement Techniques Association (2018).

[6] C. H. Schmidt, S. F. Razavi, T. F. Eibert and Y. Rahmat-Samii. "Phaseless spherical near-field antenna measurements for low and medium gain antennas". Advances in Radio Science. vol. 8. pp. 43-48, Sep. 2010.

[7] J. Fernandez Alvarez, S. Pivnenko, and O. Breinbjerg. "Probe-Corrected Phaseless Planar Near-Field Antenna Measurements at $60 \mathrm{GHz}$. Proceedings of the 37th Annual Symposium of the Antenna Measurement Techniques Association (2015).

[8] S. F. Razavi and Y. Rahmat-Samii, "A new look at phaseless planar nearfield measurements: limitations, simulations, measurements, and a hybrid solution", IEEE Antennas and Propagation Magazine, vol. 49, no. 2, pp. 170-178, April 2007.

[9] Y. Alvarez, F. Las-Heras and M. R. Pino, "The Sources Reconstruction Method for Amplitude-Only Field Measurements," IEEE Transactions on Antennas and Propagation, vol. 58, no. 8, pp. 2776-2781, Aug. 2010.

[10] S. F. Razavi and Y. Rahmat-Samii, "On the uniqueness of planar nearfield phaseless antenna measurements based on two amplitude-only measurements", 2008 IEEE Antennas and Propagation Society International Symposium, San Diego, CA, 2008, pp. 1-4.

[11] J. E. Hansen, Spherical Near-Field Antenna Measurements. London, U.K.: Peter Peregrinus, 1988.

[12] C. Cappellin, "Antenna Diagnostics for Spherical Near-Field Antenna Measurements" (PhD thesis). Technical University of Denmark (DTU), Kgs. Lyngby, Denmark, 2008.

[13] D. M. Kerns, "Plane-wave scattering-matrix theory of antennas and antenna-antenna interactions" in NBS Monograph, Washington, DC:U.S. Govt. Printing Office, vol. 162, June 1981.

[14] E. J. Candès, X. Li and M. Soltanolkotabi, "Phase Retrieval via Wirtinger Flow: Theory and Algorithms", IEEE Transactions on Information Theory, vol. 61, no. 4, pp. 1985-2007, April 2015. 